\title{
Stringy excitation and role of UV gluons in lattice QCD
}

\author{
Hiroshi Ueda*, Takahiro M. Doi, Sho Fujibayashi, Shoichiro Tsutsui, Takumi Iritani, \\ Hideo Suganuma \\ Department of Physics, Kyoto University, Kitashirakawaoiwake, Sakyo, Kyoto 606-8502, Japan \\ E-mail: uedadruby.scphys.kyoto-u.ac.jp
}

\begin{abstract}
Using SU(3) quenched lattice QCD, we study ground-state and low-lying even-parity excitedstate potentials of quark-antiquark systems in terms of the gluon-momentum component in the Coulomb gauge. By introducing UV-cut in the gluon-momentum space, we investigate the "UVgluon sensitivity" of the ground-state and excited-state potentials and the stringy excitation quantitatively. Even after cutting off high-momentum gluon component above $1.5 \mathrm{GeV}$, the IR part of the ground-state potential is almost unchanged. On the other hand, the change of excitedstate potential is more significant by the cut of UV-gluons. However, even after the removal of UV-gluons, the magnitude of the low-lying gluonic excitation remains to be of the order of $1 \mathrm{GeV}$.
\end{abstract}

The 30 International Symposium on Lattice Field Theory - Lattice 2012,

June 24-29, 2012

Cairns, Australia

${ }^{*}$ Speaker. 


\section{Introduction}

Unlike QED, quantum chromodynamics (QCD) forms a color-electric flux-tube between the quark and the antiquark in mesonic systems, and this one-dimensional squeezing of the colorelectric field leads to a linear confinement potential in the infrared region [四. Actually, apart from the color-Coulomb energy around quarks, the flux-tube formation has been observed in lattice QCD both for $Q \bar{Q}$ [[]] and $3 Q$ systems [园, 田, []].

In the flux-tube picture of hadrons, which is idealized as the string picture in the infrared region, one can expect "stringy excitations" of hadrons, as shown in Fig.1. This stringy mode is non-quark-origin excitation, and therefore it can be regarded as a gluonic excitation. Such a gluonic-excited state would be interpreted as hybrid hadrons $(q \bar{q} G$ and $q q q G)$, which are interesting hadrons beyond the quark-model framework [四].
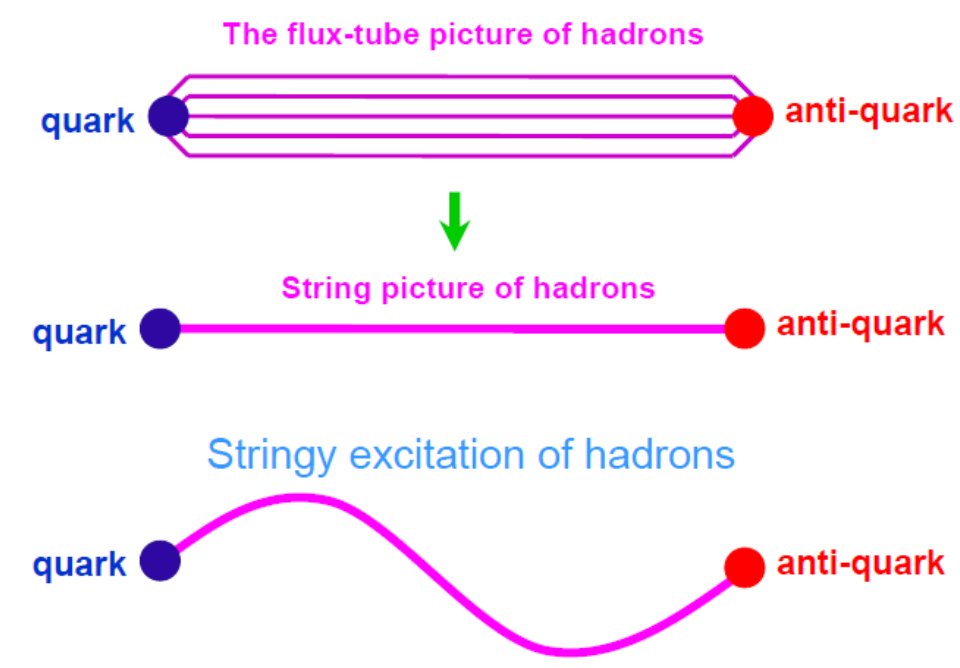

Figure 1: Schematic illustration of the stringy excitation of hadrons. The flux-tube picture of hadrons is idealized as the string picture in the infrared region, which is expected to allow "stringy excitations" of hadrons. Since this stringy mode is non-quark-origin excitation, it can be regarded as a gluonic excitation.

In lattice QCD, from a detailed Wilson-loop analysis, the excited-state potentials and the glu-


[ㅁ, 圆]. For simpler $Q \bar{Q}$ systems, the behavior of the gluonic excitation is almost consistent with the string excitation in infrared region, in spite of a significant difference at the small distance [ 6$]$.

In the previous work, IR/UV-gluon contribution to the ground-state potential has been studied [ㅇ, 미], and the confinement force is found to be almost unchanged even after the cut of highmomentum gluon components above $1.5 \mathrm{GeV}[Q, \mathbb{Q}, \mathbb{0}]$ in the Landau gauge. This means that the confinement property is insensitive to UV gluons.

In this work, we study not only ground-state potential but also low-lying even-parity excitedstate potentials of $Q \bar{Q}$ systems in terms of gluon momentum component in the Coulomb gauge. By introducing UV-cut in three-dimensional gluon-momentum space, we study the UV-gluon contribution to excited-state potentials and stringy excitations. 


\section{Lattice formulation}

\subsection{Formalism to extract excited-state potentials in lattice QCD}

We present the formalism to extract the excited-state potential [ [ $[$, [] for the spatially-fixed $Q \bar{Q}$ system. We denote the $n$th eigen-state of the QCD Hamiltonian $H$ by $|n\rangle$,

$$
H|n\rangle=V_{n}|n\rangle .
$$

Here, $V_{n}$ denotes $n$th excited-state potential, and 0th eigen-state means the ground-state. Consider arbitrary independent $Q \bar{Q}$ states $\left|\phi_{k}\right\rangle(k=0,1,2 \ldots)$. Generally, each $Q \bar{Q}$ state $\left|\phi_{k}\right\rangle$ can be expressed by a linear combination of the $Q \bar{Q}$ physical eigen-states:

$$
\left|\phi_{k}\right\rangle=c_{0}^{k}|0\rangle+c_{1}^{k}|1\rangle+c_{2}^{k}|2\rangle+\ldots .
$$

The Euclidean-time evolution of the $Q \bar{Q}$ state $\left|\phi_{k}(t)\right\rangle$ is expressed with the operator $e^{-H t}$, which corresponds to the transfer matrix in lattice QCD. The overlap $\left\langle\phi_{j}(T) \mid \phi_{k}(0)\right\rangle$ is given by the Wilson loop $W_{T}^{j k}$, sandwiched by initial state $\phi_{k}$ at $t=0$ and final state $\phi_{j}$ at $t=T$, and is expressed in the Euclidean Heisenberg picture as

$$
\begin{aligned}
W_{T}^{j k} & \equiv\left\langle\phi_{j}(T) \mid \phi_{k}(0)\right\rangle=\left\langle\phi_{j}|W(T)| \phi_{k}\right\rangle=\left\langle\phi_{j}\left|e^{-H T}\right| \phi_{k}\right\rangle \\
& =\sum_{m=0}^{\infty} \sum_{n=0}^{\infty} \bar{c}_{m}^{j} c_{n}^{k}\left\langle m\left|e^{-H T}\right| n\right\rangle=\sum_{n=0}^{\infty} \bar{c}_{n}^{j} e^{-V_{n} T} c_{n}^{k},
\end{aligned}
$$

with the complex-conjugate notation of $\bar{c}_{n}^{j} \equiv\left(c_{n}^{j}\right)^{*}$. This is a basic relation between Wilson loops and potentials. By introducing the matrices $C$ and $\Lambda_{T}$ such that

$$
C^{n k}=c_{n}^{k}, \quad \Lambda_{T}^{m n}=e^{-V_{n} T} \delta^{m n},
$$

this relation can be rewritten as

$$
W_{T}=C^{\dagger} \Lambda_{T} C
$$

In general, $C$ is not a unitary matrix, and depends on the choice of $\left|\phi_{k}\right\rangle$. Using this relation, we extract the potentials $V_{n}(n=0,1,2 \cdots)$ from the Wilson loop $W_{T}$. Consider the following combination:

$$
W_{T}^{-1} W_{T+1}=\left\{C^{\dagger} \Lambda_{T} C\right\}^{-1} C^{\dagger} \Lambda_{T+1} C=C^{-1} \operatorname{diag}\left(e^{-V_{0}}, e^{-V_{1}}, e^{-V_{2}}, \ldots\right) C .
$$

Then, $e^{-V_{n}}$ can be obtained as the eigen-values of the matrix $W_{T}^{-1} W_{T+1}$. In fact, they are the solutions of the secular equation,

$$
\operatorname{det}\left\{W_{T}^{-1} W_{T+1}-t \mathbf{1}\right\}=\prod_{n}\left(e^{-V_{n}}-t\right)=0 .
$$

In this way, the potentials $V_{n}(n=0,1,2, \ldots)$ can be obtained from the Wilson loop matrix, $W_{T}^{-1} W_{T+1}$.

In the practical calculation, we prepare gauge-invariant $Q \bar{Q}$ states $\left|\phi_{k}\right\rangle$ composed by fat-links obtained with APE smearing method [U]], and calculate many Wilson loops sandwiched by various combination of initial state $\left|\phi_{k}\right\rangle$ and final state $\left|\phi_{j}\right\rangle$. By solving the secular equation Eq. (2.8) within a truncated dimension, ground-state and excited-state potentials can be obtained. 


\subsection{Discrete Fourier transformation and UV-cut of gluon momentum components}

In this subsection, we consider the three-dimensional Fourier transformation of the link-variable $U_{\mu}(x) \in \mathrm{SU}(3)$ on a periodic lattice of size $L^{4}$, and introduce UV-cut in three-dimensional momentum space [Q, 四]. For the argument on the gluon momentum, gauge fixing is generally needed. For the comparison with continuum QCD, the suitable gauge to be taken on lattice would be the Landau or the Coulomb gauge, where the gauge field tends to be continuous.

Here, we consider link-variables fixed in the Coulomb gauge, because spatial gauge-field fluctuation is strongly suppressed. The Coulomb gauge has a global definition to minimize the "total amount of the spatial gauge-field fluctuation",

$$
R \equiv \int d^{3} x \operatorname{tr}\left\{A_{i}(\vec{x}, t) A_{i}(\vec{x}, t)\right\}=\frac{1}{2} \int d^{3} x A_{i}^{a}(\vec{x}, t) A_{i}^{a}(\vec{x}, t)
$$

The Coulomb gauge has a physical meaning that it maximally suppresses artificial fluctuation stemming from gauge degrees of freedom for spatial gluons. In lattice $\mathrm{QCD}$, the Coulomb gauge fixing is expressed in terms of link-variable and is defined by the maximization of

$$
R_{\text {latt }} \equiv \sum_{\vec{x}} \sum_{i} \operatorname{Re} \operatorname{tr} U_{i}(\vec{x}, t) .
$$

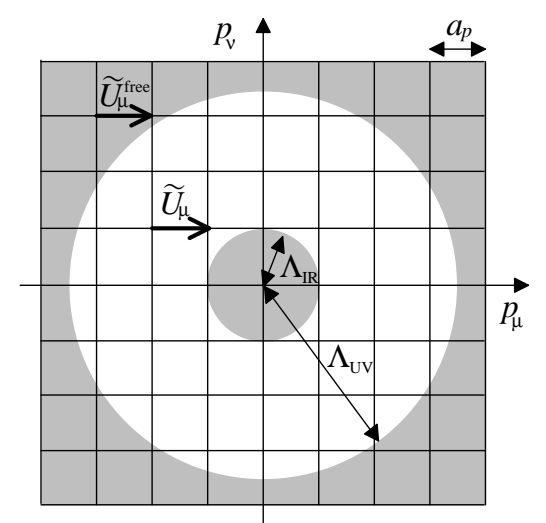

Figure 2: A schematic figure of UV-cut with $\Lambda_{\mathrm{UV}}$ and IR-cut with $\Lambda_{\mathrm{IR}}$ on momentum-space lattice, of which spacing is given by $a_{p} \equiv 2 \pi /(L a)$. The momentum-space link-variable $\tilde{U}_{\mu}(p)$ is replaced by the free-field link-variable $\tilde{U}_{\mu}^{\text {free }}(p)=\delta_{p 0}$ in the shaded cut regions.

Now, we perform the three-dimensional discrete Fourier transformation of the link-variable $U_{\mu}(x) \in \mathrm{SU}(3)$, and define the "momentum-space link-variable":

$$
\tilde{U}_{\mu}(\vec{p}, t) \equiv \frac{1}{L^{3}} \sum_{\vec{x}} U_{\mu}(\vec{x}, t) \exp (i \vec{p} \cdot \vec{x}) .
$$

We introduce "UV-cut" in the momentum space. Outside the cut, $\tilde{U}_{\mu}(\vec{p}, t)$ is replaced by 0 , since momentum-space link-variable is $\tilde{U}_{\mu}^{\text {free }}(p)=\delta_{p 0}$ in the free-field case of $U_{\mu}^{\text {free }}(x) \equiv 1$. (See Fig.1.) We define "UV-cut momentum-space link-variable":

$$
\tilde{U}_{\mu}^{\Lambda}(\vec{p}, t) \equiv\left\{\begin{array}{lll}
\tilde{U}_{\mu}(\vec{p}, t) & \text { for } & |\vec{p}| \leq \Lambda_{\mathrm{UV}} \\
0 & \text { for } & |\vec{p}|>\Lambda_{\mathrm{UV}}
\end{array}\right.
$$


By the three-dimensional inverse Fourier transformation

$$
U_{\mu}^{\prime}(\vec{x}, t) \equiv \sum_{\vec{p}} \tilde{U}_{\mu}^{\Lambda}(\vec{p}, t) \exp (-i \vec{p} \cdot \vec{x}),
$$

and $\mathrm{SU}(3)$ projection by maximizing

$$
\operatorname{Re} \operatorname{tr}\left\{U_{\mu}^{\Lambda}(\vec{x}, t) U_{\mu}^{\prime \dagger}(\vec{x}, t)\right\},
$$

we obtain "UV-cut (coordinate-space) link-variable":

$$
U_{\mu}^{\Lambda}(\vec{x}, t) \in \mathrm{SU}(3)
$$

Using the UV-cut link-variable $U_{\mu}^{\Lambda}(x)$ instead of $U_{\mu}(x)$, we calculate many Wilson loops $W_{T}^{i k}$ sandwiched by various combination of initial state $\left|\phi_{k}\right\rangle$ and final state $\left|\phi_{j}\right\rangle$ Note here that the UV-cut should be introduced also to $U_{4}(x)$. Otherwise, the QCD Hamiltonian is not changed, so that the potentials $V_{n}$ are not changed at all.

\section{Ground-state and excited-state $Q \bar{Q}$ potentials and gluonic excitation energy}

In this section, we show the lattice QCD results of the ground-state/excited-state potentials and gluonic excitation energy in $Q \bar{Q}$ systems with or without UV-cut. Our numerical simulation is performed with isotropic plaquette gauge action with $\beta=6.0$ at the quenched level. The lattice size is $16^{4}$, and the periodic boundary condition is imposed. This lattice QCD condition corresponds to the (coordinate-space) lattice spacing $a \simeq 0.104 \mathrm{fm}$, and the momentum-space lattice spacing $a_{p} \equiv 2 \pi /(L a) \simeq 0.74 \mathrm{GeV}$. We use 100 gauge configurations, and average all the parallel-translated Wilson loops in each configuration.
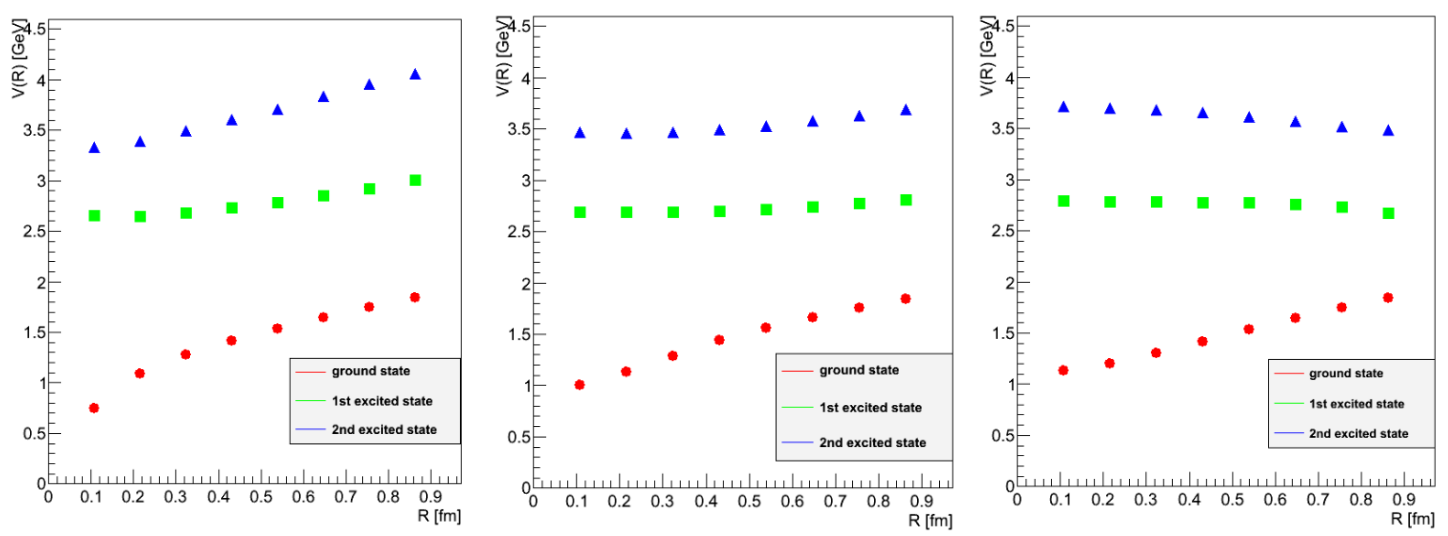

Figure 3: Ground-sate and even-parity excited-state potentials in $Q \bar{Q}$ systems with or without UV-cut plotted against the interquark distance $R$. The left panel shows the result without UV-cut. The middle and right panels show the results with the UV-cut of $\Lambda_{\mathrm{UV}}=3 a_{p} \simeq 2.2 \mathrm{GeV}$ and $\Lambda_{\mathrm{UV}}=2 a_{p} \simeq 1.5 \mathrm{GeV}$, respectively. The circle symbol denotes the ground-state potential. The square and the triangle symbols denote the evenparity excited-state potentials.

For simplicity, we only consider even-parity exited-state potentials in this paper. We prepare the $Q \bar{Q}$ state $\left|\phi_{k}\right\rangle(k=0,1,2,3)$ composed by the "fat-links" obtained by the APE smearing method 
[U]] with the smearing parameter $\alpha=2.3$ and the iteration number of $N_{\mathrm{smr}}=0,8,16,24$. Note that only even-parity components can be obtained by this parity-invariant procedure.

\subsection{Ground-state and excited-state $Q \bar{Q}$ potentials with UV cut}

Figure $[3$ shows ground-state and excited-state potentials in $Q \bar{Q}$ systems with or without UVcut of gluon fields. In the original no UV-cut case, the IR slopes of ground-state and excited-state potentials are almost the same, as was indicated by the previous lattice studies [ [G]. This means the same confinement force in the infrared region.

By the cut of UV-gluon above $\Lambda_{\mathrm{UV}}=3 a_{p} \simeq 2.2 \mathrm{GeV}$, the short-distance Coulomb part proportional to $1 / r$ reduces in ground-state potential. In the case of $\Lambda_{\mathrm{UV}}=2 a_{p} \simeq 1.5 \mathrm{GeV}$, the shortdistance Coulomb part disappears in the ground-state potential. These tendencies are consistent with the previous studies [Q, 四].

On the other hand, the shape of the excited-state potential is largely changed by the UV-cut of gluon fields for $\Lambda_{\mathrm{UV}}=1.5,2.2 \mathrm{GeV}$, while the ground-state potential is not so changed except for the short distance. As a caution, the physical size of our lattice is rather small, and the true IR slope of the excited-state is expected to be unchanged, because no change is found in the ground-state potential, which gives a lower bound of $V_{n}$ in the infrared region. In any case, the change of the excited-potential is more significant than that of the ground-state potential by the UV-cut of gluons.

In the string picture, this result seems to be natural as mentioned below. For the stringy-excited state as shown in Fig.1, there is a typical wavelength proportional to the interquark distance $R$, and this wavelength is smaller for higher excitation mode. Then, we expect a significant influence of the removal of UV-gluons for the stringy mode, when the UV-cut length $1 / \Lambda_{U V}$ becomes larger than the typical wavelength of the stringy excitation. In fact, the effect of UV-gluon cut would be larger for higher excitation. Our lattice QCD results seem to be qualitatively consistent with this tendency.

\subsection{Gluonic excitation energy with UV cut}

The gluonic excitation energy defined by the difference, $V_{n}-V_{0}$, is shown in Fig.G. Roughly, even after the removal of UV-gluons, the magnitude of gluonic excitation is approximately unchanged, and the low-lying gluonic excitation remains to be of the order of $1 \mathrm{GeV}$.
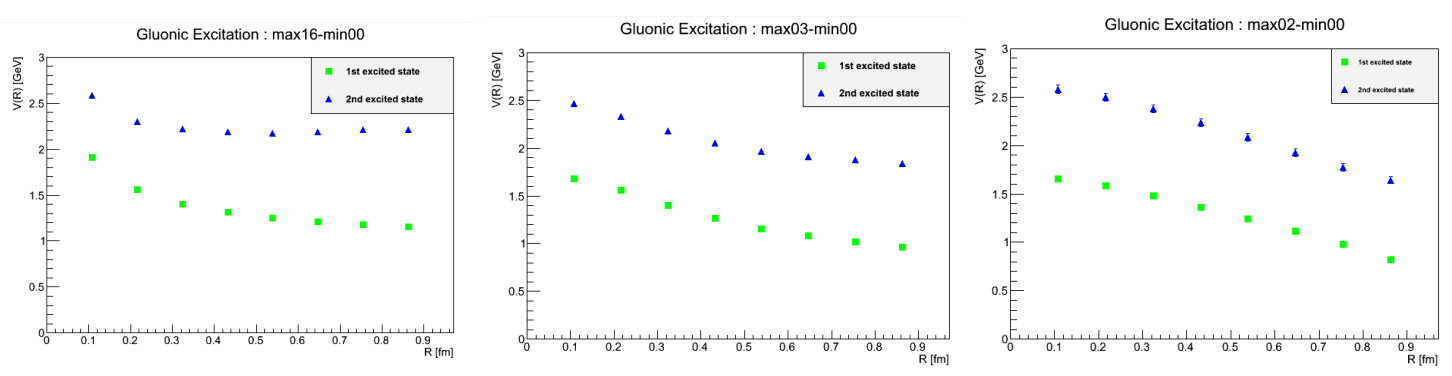

Figure 4: The gluonic excitation energy defined by the difference between the ground-state and the excitedstate $Q \bar{Q}$ potentials, $\Delta E_{n}=V_{n}-V_{0}$. The left panel shows no UV-cut case. The middle and right panels show the results with the $\mathrm{UV}$-cut of $\Lambda_{\mathrm{UV}}=3 a_{p} \simeq 2.2 \mathrm{GeV}$ and $\Lambda_{\mathrm{UV}}=2 a_{p} \simeq 1.5 \mathrm{GeV}$, respectively. 


\section{Summary and concluding remarks}

In this study, we have studied ground-state and low-lying even-parity excited-state potentials of quark-antiquark systems in terms of the gluon momentum component in the Coulomb gauge using SU(3) quenched lattice QCD. By introducing UV-cut in the gluon momentum space, we study the "UV-gluon sensitivity" of the potentials and the stringy excitation. Even after cutting off high-momentum gluon component above $1.5 \mathrm{GeV}$, the IR part of the ground-state potential is almost unchanged. On the other hand, the change of excited-state potential is more significant by the cut of UV-gluons. However, the magnitude of the low-lying gluonic excitation remains to be of the order of $1 \mathrm{GeV}$ after the removal of UV-gluons.

As a next step, we will investigate the odd-parity excited-state potential, by using non-symmetric state operators of $|\phi\rangle_{k}$. In this work, we use the Coulomb gauge fixing, however, to remove gauge artifact completely, it would be also interesting to apply the gauge-invariant expansion in terms of the Dirac mode [ए2].

\section{Acknowledgements}

H.S. is supported by the Grant for Scientific Research [(C) No.23540306, Priority Areas "New Hadrons" (E01:21105006)] and T.I. is supported by a Grant-in-Aid for JSPS Fellows [No. 23-752] from the Ministry of Education, Culture, Science and Technology (MEXT) of Japan. This work is supported by the Global COE Program, "The Next Generation of Physics, Spun from Universality and Emergence". The lattice QCD calculation has been done on NEC-SX8R at Osaka University.

\section{References}

[1] Y. Nambu, Phys. Rev. D10 (1974) 4262.

[2] G. S. Bali, K. Schilling and C. Schlichter, Phys. Rev. D51 (1995) 5165 [hep-lat/9409005].

[3] H. Ichie, V. Bornyakov, T. Streuer and G. Schierholz, Nucl. Phys. A721 (2003) 899 [hep-lat/0212036].

[4] T.T. Takahashi, H. Suganuma, H. Ichie, H. Matsufuru and Y. Nemoto, Nucl. Phys. A721 (2003) 926.

[5] V.G. Bornyakov et al. [DIK Collaboration], Phys. Rev. D70 (2004) 054506 [hep-lat/0401026].

[6] K. J. Juge, J. Kuti and C. Morningstar, Phys. Rev. Lett. 90 (2003) 161601 [hep-lat/0207004].

[7] T. T. Takahashi and H. Suganuma, Phys. Rev. Lett. 90 (2003) 182001 [hep-lat/0210024].

[8] T. T. Takahashi and H. Suganuma, Phys. Rev. D70 (2004) 074506 [hep-lat/0409105].

[9] A. Yamamoto and H. Suganuma, Phys. Rev. Lett. 101 (2008) 241601 [arXiv:0808.1120 [hep-lat]].

[10] A. Yamamoto and H. Suganuma, Phys. Rev. D79 (2009) 054504 [arXiv:0811.3845 [hep-lat]].

[11] M. Albanese et al. [APE Collaboration], Phys. Lett. B192 (1987) 163.

[12] S. Gongyo, T. Iritani and H. Suganuma, Phys. Rev. D86 (2012) 034510 [arXiv:1202.4130 [hep-lat]]. 